\title{
Proactive and Predictive Maintenance Strategies and Application for Instrumentation \& Control in Oil \& Gas Industry
}

\author{
Ngu Kie Ming ${ }^{1 *}$, Nicholas Philip ${ }^{1}$, Shafishuhaza Sahlan² \\ ${ }^{1}$ Asset Integrity Department, PETRONAS LNG Complex, Tanjung Kidurong, 97000 Bintulu, Sarawak, Malaysia \\ ${ }^{2}$ Faculty of Electrical Engineering, Universiti Teknologi Malaysia, 81310 UTM Skudai, Johor, Malaysia \\ *Corresponding Author
}

DOI: https://doi.org/10.30880/ijie.2019.11.04.013

Received 24 April 2019; Accepted 1 August 2019; Available online 5 September 2019

\begin{abstract}
Instrumentation \& Control Systems have gone through various revolutions in the oil and gas industry. The start of the industry was based on pneumatic controllers, operating with instrument air or instrument gas at 0.2 to 1.0 barg and the Instrument Protective System (IPS) was relay based. This system provided almost zero information or data that can be used to predict failure and had a higher unrevealed failure. The next phase of instrumentation was migration into the electronics era. This allowed for the migration from a pneumatic system to the application of electronics field device operation on 4-20mA connected to Distributed Control System (DCS) and IPS. Further development of $4-20 \mathrm{~mA}$ communication protocol allowed for the development of digital superimpose communication called Highway Addressable Remote Transducer (HART). The data that is transmitted in HART protocol provides sufficient data and information to predict the health and functionality of the instrumentation. Changes in the maintenance philosophy from reactive maintenances to proactive or predictive maintenances, resulted in reduced downtime by scheduling maintenance to optimize the working window. This work process allows for greater productivity of assets, both human and capital. Dashboards are developed and utilised to alert fault detection and loss of redundancy, whereby data is relayed from DCS and IPS to a web-based system. Available tools being developed in the industry and application of IoT within the industry allows for realtime field device health monitoring and an engine to predict deviations. The strength and weakness of the various proactive or predictive maintenance strategies are compared and summarized, including scope for future research and application in the industry.
\end{abstract}

Keywords: Distributed Control System, Proactive Maintenance, Predictive Maintenance, HART, web-based system, Instrument Protective System

\section{Introduction}

The oil \& gas industry is a challenging and hazardous industry. To fuel the energy requirements for the world economy, the challenge in exploration and production of oil and gas is getting tougher. As the number of industry players and production increases, so does the risk. Therefore, safety is now front and center of the industry. This is noticeable in almost all major oil companies where there is a strong industrial culture of workplace safety with more advanced technologies to monitor and prevent injuries and accidents, including process safety incidents.

Figure 1 shows a diagram on Layer of Protection in the process industry, especially applicable to the oil and gas industry. This diagram is also known as the onion layer model and can also be shown in the form of a bow-tie. The process condition is maintained at a safe operating limit within the process control layer. Control layer is implemented in the Distributed Control System (DCS). 


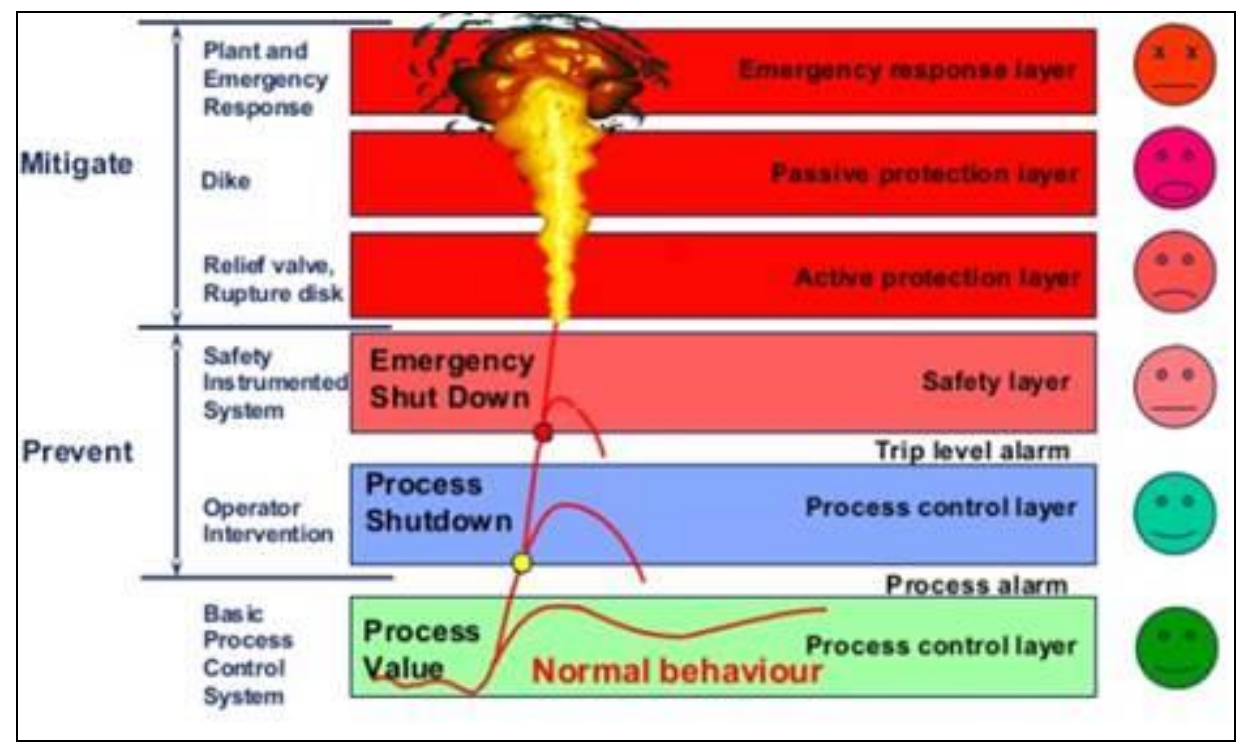

Fig. 1 - Safety through Layers of Protection

The typical control in the oil and gas industry is based on the PID controller, with the application of various types of control schemes as provided in Figure 2. If the process value goes out of control from the normal behaviour, the process will continue to increase and move out of the normal operating condition. This condition will activate a process alarm implemented with the controller or as independent measurement, with the alarm activated in the DCS. The next layer of protection will be a process shutdown condition, which will initiate a process shutdown. If the process continues to go out of control and all previous layers failed to mitigate, the next protection layer is called the safety layer. These protection layers are implemented in dedicated safety systems known as Safety Instrumented Systems (SIS), which are designed to comply with IEC 61508 and IEC 61511 requirements. The design criteria of the system is to meet rigorous requirements such as scan time of $300 \mathrm{~ms}$, no single point of failure, redundancy requirements, cripple mode operation requirements and diagnostic requirements.

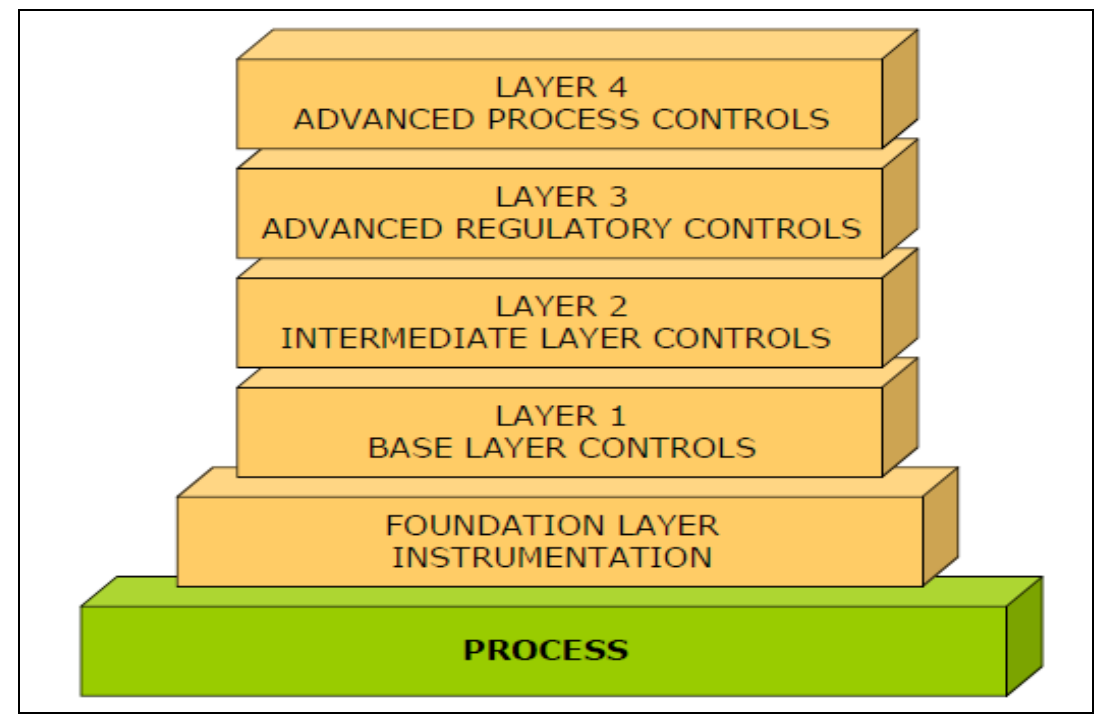

Fig. 2 - Control Scheme Layers

All the layers explained are known as preventive layers and all these are implemented in instrumentation $\&$ control systems. The next layers under mitigate are related to mechanical under active protection and passive protection. The last layer to mitigate the emergency condition is human actions under emergency response layer. As the process condition continues to break through the layers, the consequences from the emergency increase greatly.

To ensure that every layer of the instrumentation is in good working condition during the lifespan of the process plant operation, a maintenance philosophy is established. Maintenance strategies and methodology have evolved over time. From visual inspection, which is the oldest, most effective and widely used method, maintenance approach is moving from planned preventive maintenance mode to proactive or predictive maintenance. Proactive/predictive maintenance has evolved to automated methods that use advanced signal processing techniques based on pattern 
recognition, using a complex algorithm based on fuzzy logic, neural network, data-driven modeling and estimation. This is also taking place in the oil \& gas industry. Instrumentation \& Control Systems have gone through various revolutions in the oil \& gas industry. The start of the industry was based on pneumatic controllers, operating with instrument air or instrument gas at 0.2 to 1.0 barg and the Instrument Protective System (IPS) was relay based. This system provided almost zero information or data that can be used to predict failure and had a higher unrevealed failure. The next phase of instrumentation was migration into the electronics era. This allowed for the migration from a pneumatic system to the application of electronics field device operation on 4-20mA connected to Distributed Control System (DCS) and IPS. A typical modern instrumentation flows from the sensors to the logic solver or systems and the output to the final element as shown in Figure 3. Further development of 4-20mA communication protocol allowed for the development of digital superimpose communication called Highway Addressable Remote Transducer (HART). The data that is transmitted in HART protocol provides sufficient data and information to predict the health and functionality of the instrumentation. Changes in the maintenance philosophy from reactive maintenances to proactive or predictive maintenances results in reduced downtime by proactively monitoring and scheduling maintenance to optimize the working window.

This work process allows for greater productivity of assets, both human and capital. Dashboards are developed and utilised to alert fault detection and loss of redundancy, whereby data is relayed from the DCS and IPS to a web-based system. Available tools being developed in the industry and application of IoT within the industry allows for real-time field device health monitoring and the engine to predict deviations. The strength and weakness of the various predictive maintenance strategies are compared and summarized, including scope for future research and application in the industry.

\subsection{Proactive/ Predictive Maintenance}

Breakdowns and equipment failures can have major economics, safety and environment impact across the oil \& gas industry. A failure in a critical compressor, for example, the refrigeration compressor in the LNG plant can result in the shutdown of an entire plant. Major rotating equipment damages can take months or even years to replace, resulting in loss of revenue. Proactive/predictive maintenance through analytics application is a technique that allows improvement to the established planned maintenance by using data to understand equipment degradation and predict failure. By understanding the equipment criticality and the risk associated with it, maintenance can be scheduled at the best possible time that reduces disruption and leads to better cost optimization. Ability to move further into digitalization and proactive/predictive analytics is made possible due to:

- Application of smart sensors into better field instrumentation with connectivity and reliability.

- Development of IoT platforms capable of analyzing large volumes of data.

- Better connectivity such as the application of wireless systems and the ease by which process operating data and equipment health can be monitored remotely.

Proactive/predictive maintenance has the most impact on the company's revenue when applied to predict the failure of high value and critical components, where the impact of any downtime is significant. As in Figure 3, proactive/predictive maintenance methodology is applied to every component of the instrument loop.

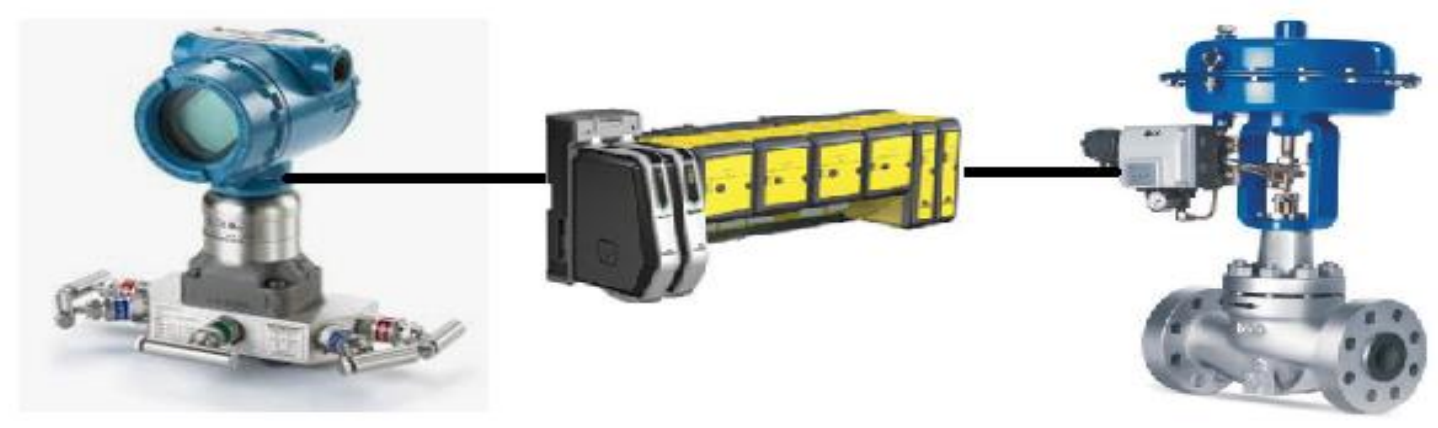

Fig. 3 - An Instrument Loop 


\subsection{Proactive/Predictive Maintenance Methods for Sensors}

To ensure the process remains within the pipe and is operated safely, key information is sent to the process operators from sensors. With further advancement in sensor technology, more data and information can be sent for diagnostics. The sensor used for process measurement such as to measure pressure, level, flow and temperature use advance transducers or transmitters operating on high-performance microprocessors. The available transmitters in the market provide advanced functionality such as $4-20 \mathrm{~mA}$ HART $^{\circledR}$ protocol, WirelessHART ${ }^{\circledR}$ (IEC 62591) and FOUNDATION ${ }^{\mathrm{TM}}$ Fieldbus capabilities.

If the transmitter self-diagnostics detects a gross transmitter failure, the analogue signal will be driven off-scale of the 4-20mA signal to alert the users. By detecting this signal at the system through either the DCS or SIS, action can be taken to alert process operators on the fault. This will help to prevent unrevealed failure and allows for proactive rectification and minimize downtime. For Safety Instrumented Function (SIF) that are in a redundant configuration such as two out of three (2oo3) to trip, the fault signal allows for an online rectification to proceed, preventing a nuisance trip. Figure 4 shows the detection level of a good operating sensor, measuring range data, over-range data and failure level.

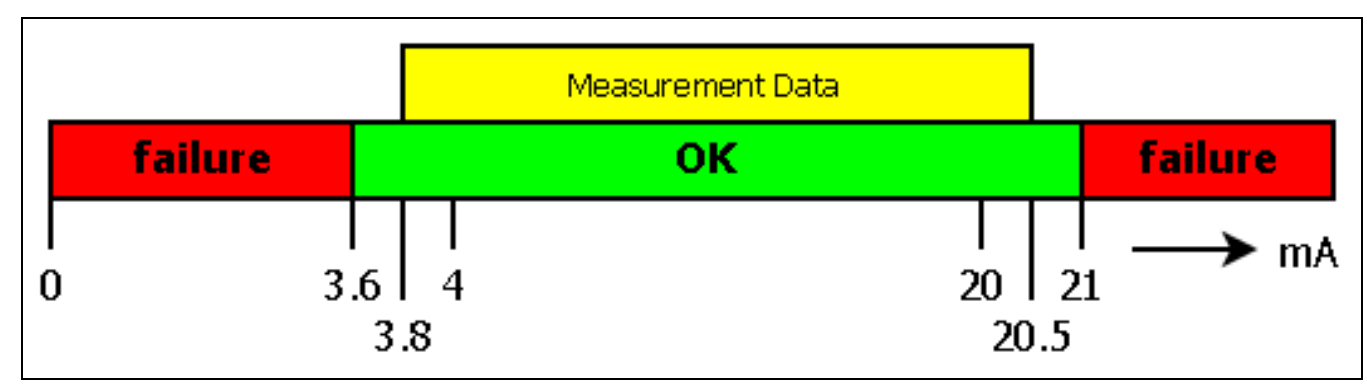

Fig. 4 - Transmitter Failure detection based on $\mathrm{mA}$ value

The settings shown in the figure is a standardize setting based on NAMUR 43. Figure 5 and 6 shows a typical logic implemented in the SIS to detect the failure alarm and activate certain actions. In Figure 5, the instrument sensor is implemented as one out of one (1oo1) configuration, when a fault is detected, fail-safe action is initiated. In Figure 6, the implementation is two out of three (2003), when a single fault happens, a fault alarm is activated and maintenance can be initiated, avoiding a shutdown of the process plant.

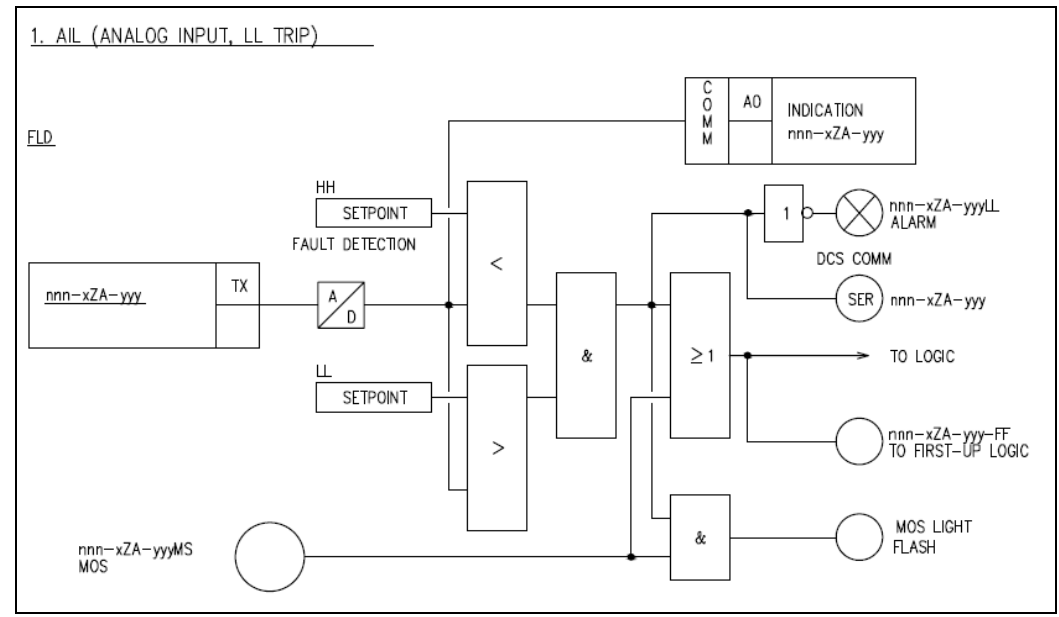

Fig. 5 - Logic Diagram with Fault Detection for 1001 


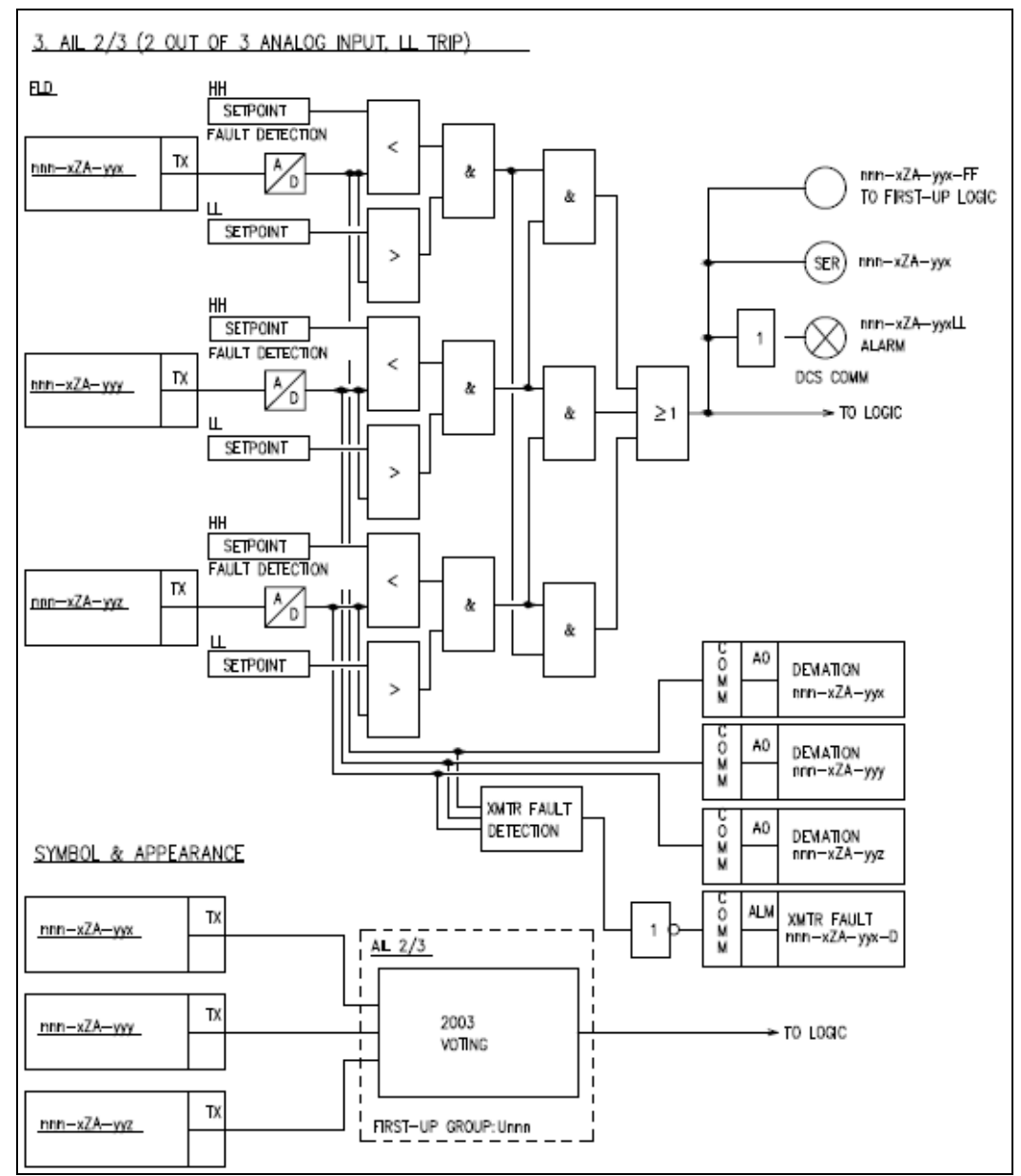

Fig. 6 - Logic Diagram with Fault Detection for 2003

HART $^{\circledR}$ field communications protocol is an extremely important technology. It is a major step in the evolution of process control and as an enabling technology that provides significant innovation in the capabilities of field instrument, allowing for the development of smart instrumentation. With $\mathrm{HART}^{\circledR}$, diagnostics data of the instrument health, condition and performance information can be sent to a central control or monitoring system. Example of data are instrument operating temperature, electronic board failure and communication failure. With the ability of the transmitter microprocessor to provide the transmitter operation condition, this allows for condition-based monitoring, for example, visual inspection for water ingress or evaluate the placement and location of the transmitter with regards to ambient temperature. Figure 7 and 8 shows some of the examples of alarms that can be detected from the HART ${ }^{\circledR}$ signal. 


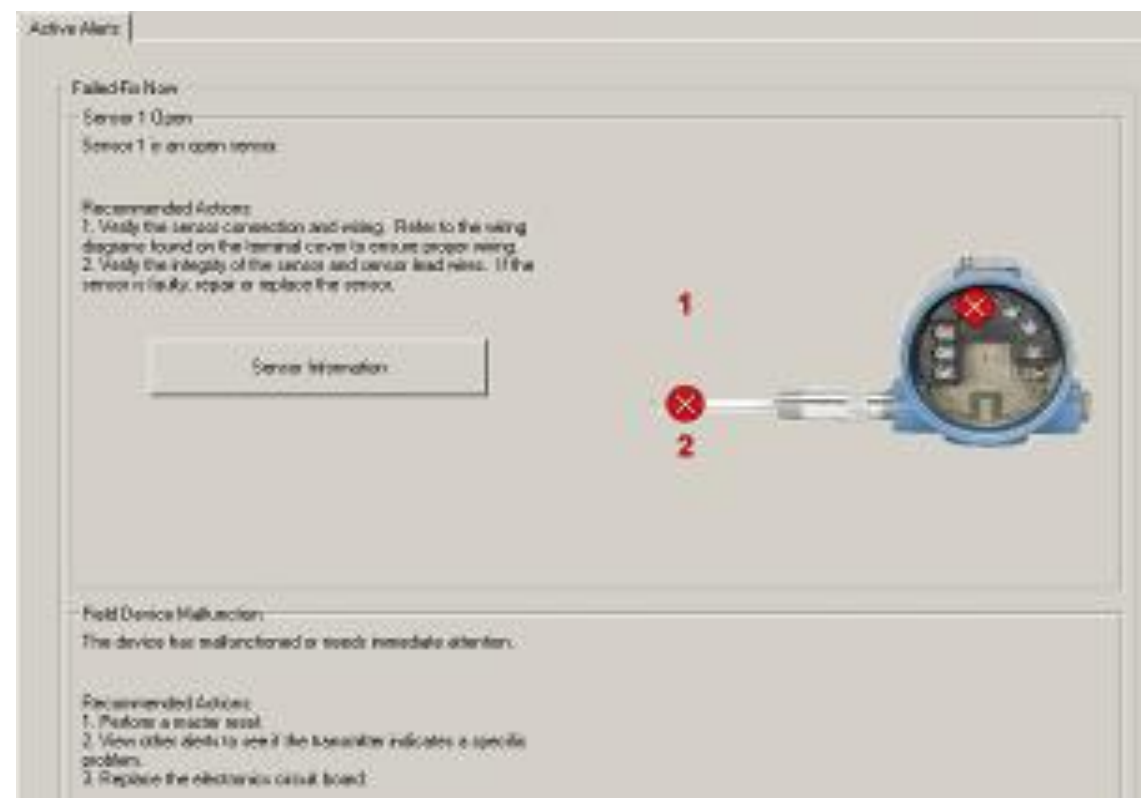

Fig. 7 - Field Device Malfunction and Recommended Action

When there are two or more sensors used to measure the same measurement, a continuous comparison between the measurements can be done to detect sensor drift, calibration issues or response. When the readings are set at certain different limits, alarms can be generated to alert the conditions. This method can be considered as providing continuous live testing of the sensors including its transducer or transmitters. Direct comparison of these sensors may not be effective and can cause a high number of nuisance alarms.

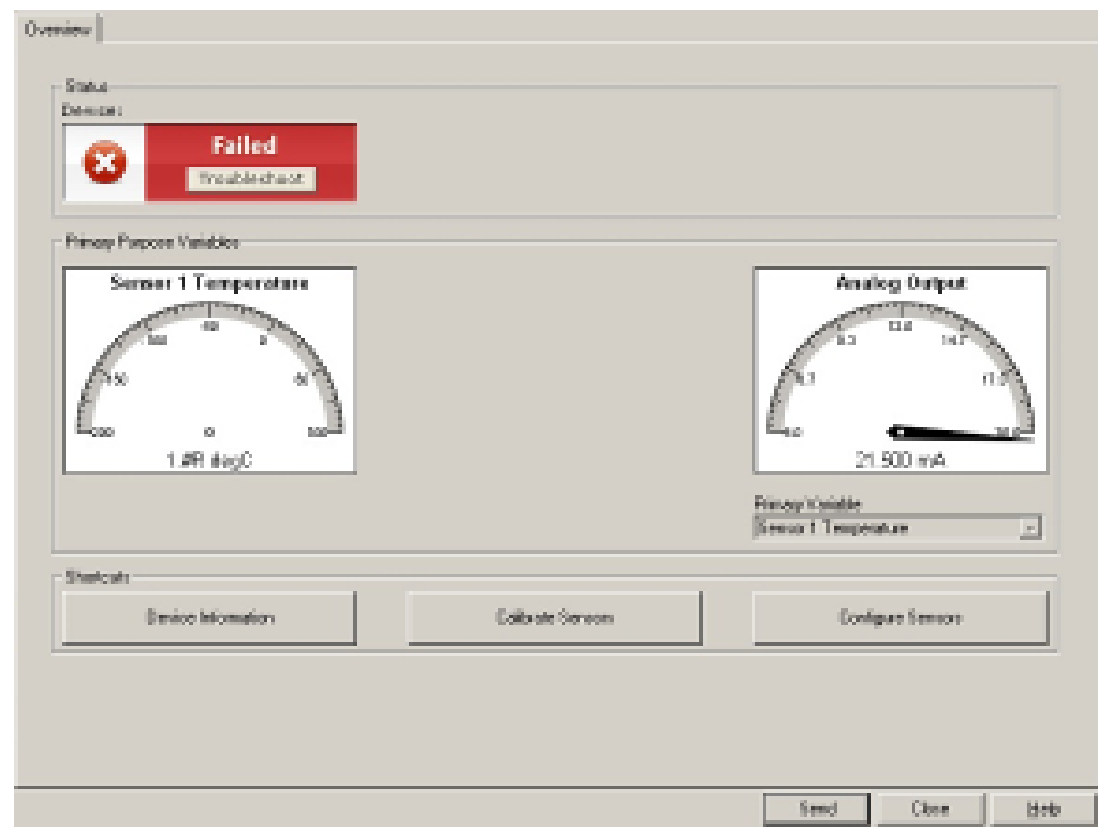

Fig. 8 Field Device Active Alert

The reason is because no two sensors can respond at the same time to the change of measurement. To address this, an algorithm can be created by sampling the reading at a periodic interval. After a certain time period, if the accumulated difference is above a certain set point, an alarm will be activated. If the differences reduce, the accumulated difference will reduce. A Deadband will automatically switch off the alarm if the accumulated reduction in difference further reduces the chattering alarm. 


\begin{tabular}{|c|c|c|c|c|c|c|}
\hline System -8 & 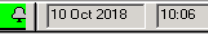 & & & & & \\
\hline \multicolumn{7}{|c|}{ 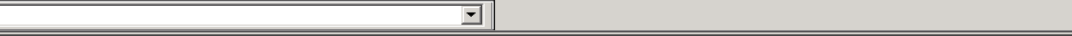 } \\
\hline MOD4 & \multicolumn{3}{|c|}{ UNIT: 2400} & \multicolumn{3}{|r|}{ WATCHDOG } \\
\hline MVC TAG & PRIMARY TAG & SECONDARY TAG & PRIMARY YQLUE & SECONDARY VQLUE & cusum & ALARM \\
\hline 424F013ABD & 424FZA_1013A & 424FZA_1013B & 233.21 & 233.44 & 0.0000 & \\
\hline 424F016F012D & 424FT012 & 424_FZA016_D & 0.0820 & -0.8704 & 0.0000 & UसACK \\
\hline 424F017F001D & 424_FT1001 & 424FZA_1017 & 6289.94 & 6250.92 & 0.0000 & \\
\hline 424F017F015D & 424_FTO15H & 424_FZA017_D & -0.0797 & -2.15 & 20.74 & \\
\hline 424F018F002D & 424_FT1002 & 424FZA_1018 & -9.60 & -3.59 & $\mathbf{0 . 0 0 0 0}$ & \\
\hline 424F019F13AD & 424_FT1019 & 424FZA_1013A & 232.94 & 233.21 & 0.0000 & \\
\hline 424F019F13BD & 424_FT1019 & 424FZA_1013B & 232.94 & 233.44 & 0.0000 & \\
\hline 424F075F077D & 424_FR075 & 424_FZA077_D & 0.0000 & -0.0860 & 2.05 & \\
\hline 424L028ABD & 424LZA028A & 424LZAO28B & 0.2353 & 0.1345 & 0.0000 & \\
\hline 424L028ACD & 424LIZA028C & 424LZA028A & -0.4706 & 0.2353 & 0.0000 & \\
\hline 424L028BCD & 424LIZAO28C & 424LZA028B & -0.4706 & 0.1345 & 0.0000 & \\
\hline 424LO30ABD & 424LZAOO3OA & 424LZAO3OB & 5.51 & 1.08 & 36.00 & \\
\hline 424LO30ACD & 424LIZAO30C & 424LZA030A & 0.0000 & 5.51 & 36.00 & \\
\hline 424LO30BCD & 424LIZAOO30C & 424LZA030B & 0.0000 & 1.08 & 0.0000 & \\
\hline 424L032ABD & 424LZA032A & 424LZA032B & 0.8067 & 3.03 & 0.0000 & \\
\hline 424LO32ACD & 424LIZAO32C & 424LZA032A & 2.89 & 0.8067 & 0.0000 & \\
\hline 424L032BCD & 424LIZAO032C & 424LZA032B & 2.89 & 3.03 & 0.0000 & \\
\hline 424LO60ABD & 424LZAO60A & 424LZA060B & -1.55 & -0.6050 & 0.0000 & \\
\hline 424LO6OACD & 424LIZAO6OC & 424LZA060A & -0.6387 & -1.55 & 0.0000 & \\
\hline 424LO6OBCD & 424LIZAO660C & 424LZA060B & -0.6387 & -0.6050 & 0.0000 & \\
\hline 424L062ABD & 424LZA062A & 424LZA062B & 1.92 & -0.1008 & 0.0000 & \\
\hline 424L062ACD & 424LIZAO62C & 424LZA062A & -1.55 & 1.92 & 0.0000 & \\
\hline 424L062BCD & 424LIZA062C & 424LZA062B & -1.55 & -0.1008 & 0.0000 & \\
\hline 424LO64ABD & 424LZA064A & 424LZAO64B & 0.7059 & 2.59 & o.0000 & \\
\hline & & & & & & \\
\hline PAGE ACK & ACK WATCHDOG & & & & & \\
\hline
\end{tabular}

Fig. 9 - Transmitter Deviation Overview

Figure 9 shows an overview of the transmitter deviation page. It shows the reading of the transmitter and the accumulated differences including the alarm. Figure 10 shows the individual comparison, faceplate and trending. This information provided help in troubleshooting such as identifying the transmitter with issues, either reading fluctuating, plateau or out of range.

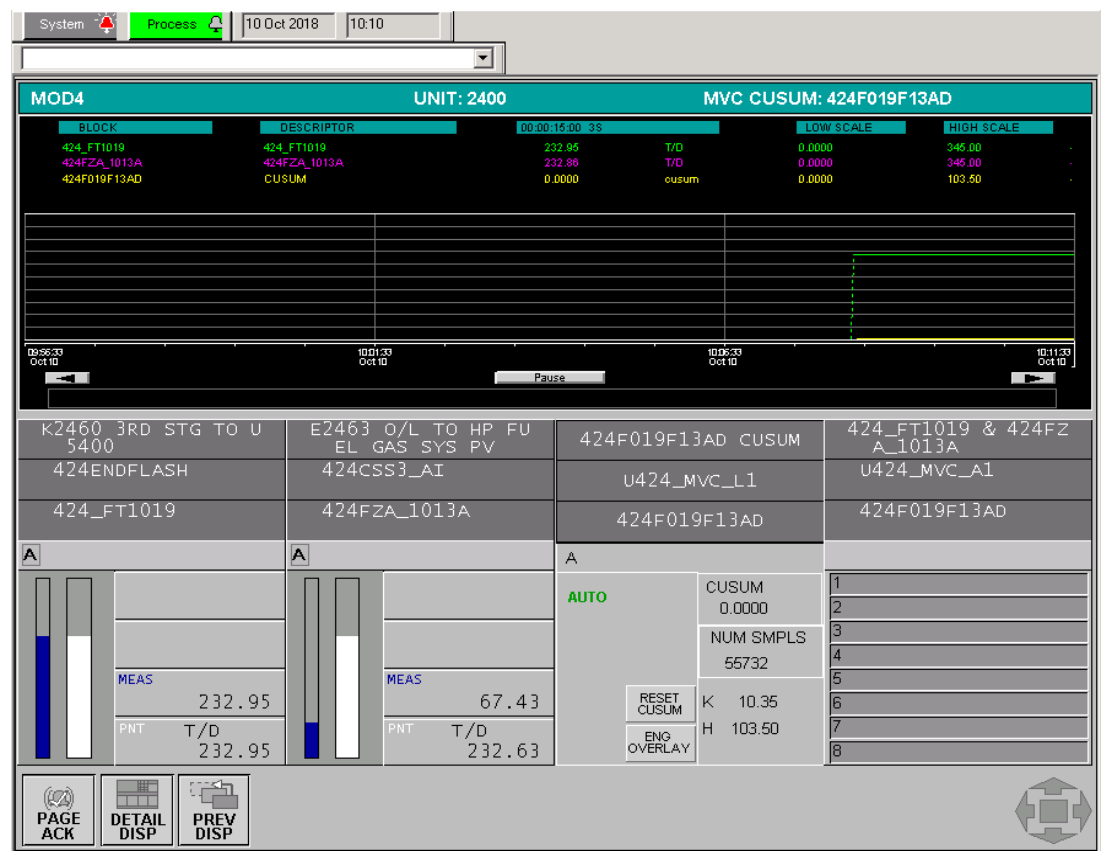

Fig. 10 - Transmitter Deviation Faceplate and Trending

\subsection{Proactive/Predictive Maintenance Methods for Systems}

Systems applied in the oil \& gas industry are designed, operated and maintained based on IEC 61508 and IEC 61511. Absolute safety can never be achieved and it is acceptable that the risk can only be reduced to a tolerable level, ALARP (As Low as Reasonably Practical). As stated in IEC 61511, SIS complies with the required safety integrity requirements specify for a SIL and the performance required for executing the SIS functions. These requirements include requirements for diagnostics. These diagnostics provide predictive information of the systems that prevent a complete failure that could lead to hazardous consequences. Extensive diagnostics covering each channel, module, control processor, I/O cards with functional circuits to quickly detect and report operational faults. This information is available in the application dashboard that allows for proper management of the information which helps to prevent an 
unnecessary shutdown of a process plant. By improving the system's availability and safety, the systems used are able to detect failures and provide the means for managing failures properly. Typically, the available diagnostics are divided as follows:

- Reference diagnostics: Comparing an operating value to a predetermined reference, such as a system specification.

- Comparison diagnostics: Comparing one component to another, such as one independent channel with other independent channels.

- Field device diagnostics: Diagnostics are extended to a systems' field device and wiring.

The systems are subjected to both internal and external faults. Internal faults are usually isolated from each component as typically system applications have redundancy as a minimum baseline. Other than redundancy, systems are also designed as Triple Modular Redundancy (TMR) or Quad. Internal faults depending on the severity may cause systems to operate as crippled mode operation until rectification is done. External faults are related to field instrument faults, power failure fault, and load or fuse faults. Figure 11 shows the related alarms for monitoring the performance of the IPS. Besides IPS, other systems health check are also monitored to ensure reliability and availability.

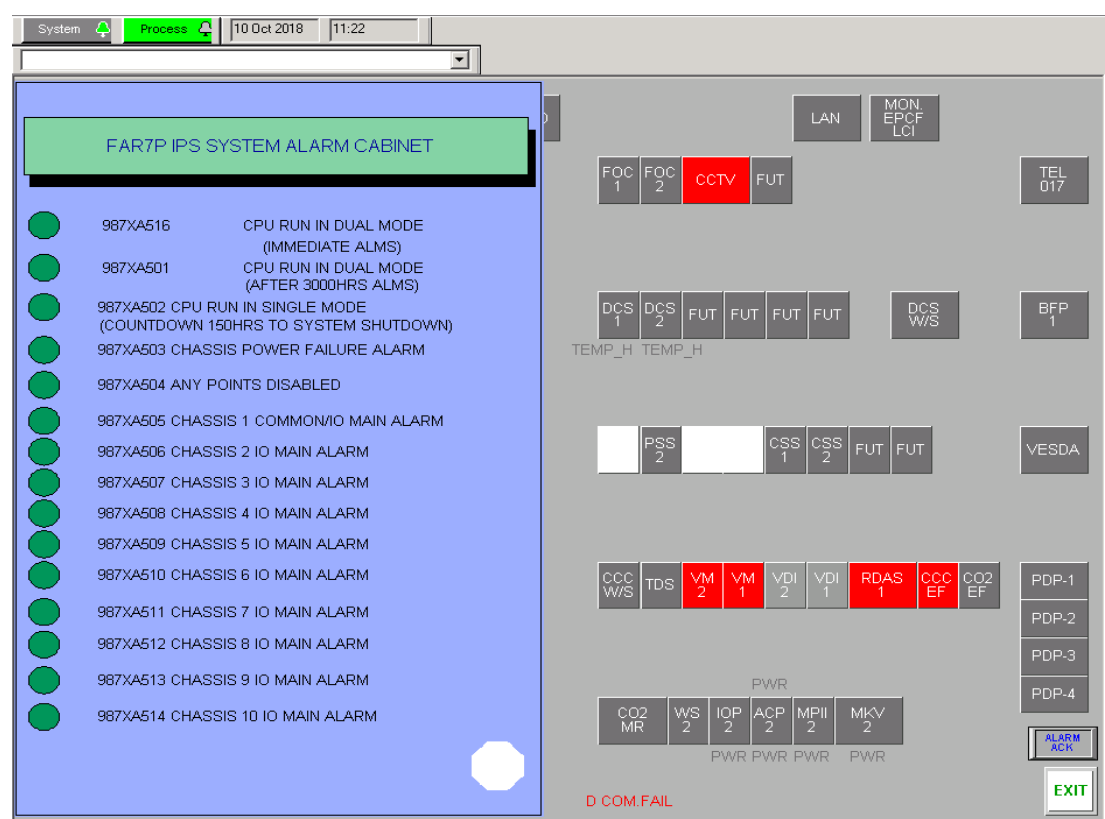

Fig. 11 - Instrument Protective System Monitoring

Similarly for other systems, related alarms and monitoring are applied. Figure 12 shows the alarms and indication of the system health that is being monitored for a custody metering system. This monitoring ensured that the custody metering system is working in accordance to the contractual agreement and any fault or failures can be initiated immediately. 


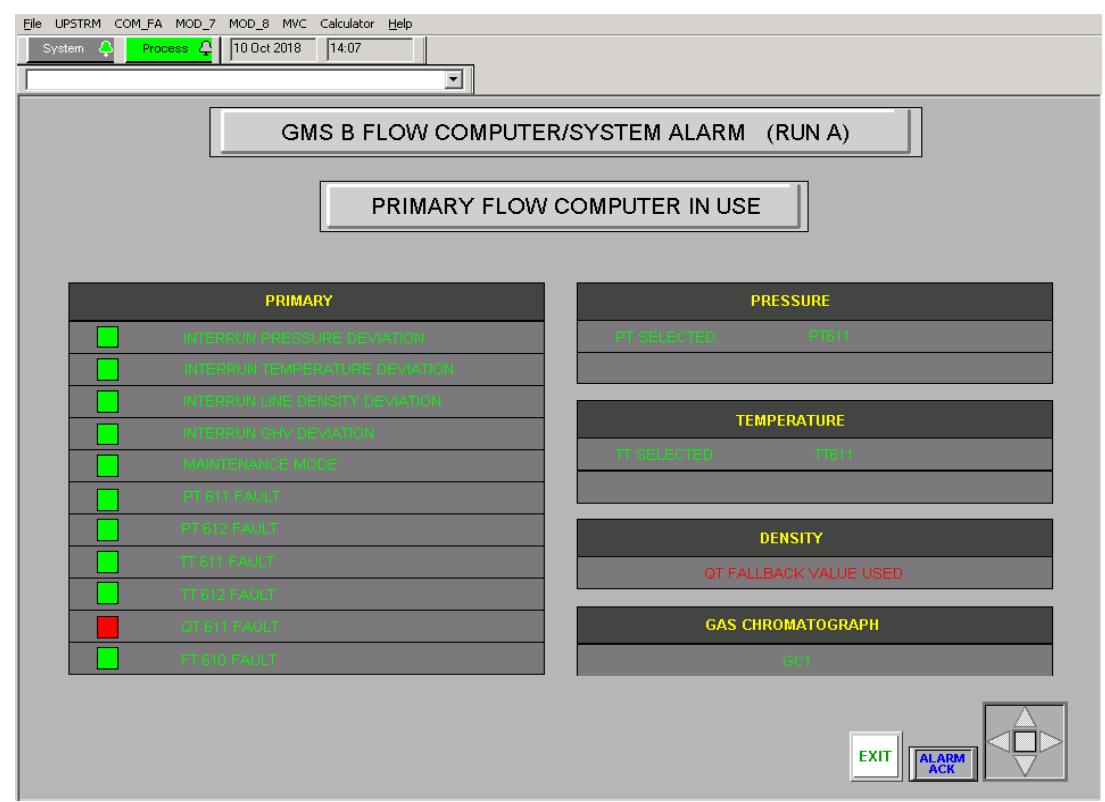

Fig. 12 - Gas Metering System Status Monitoring

Figure 13 shows a Boiler Management System (BMS) health status monitoring. Among the status being monitored are power supply redundancy, communication redundancy, cabinet temperature, fan operational, $\mathrm{I} / \mathrm{O}$ card failure and system internal failures. These critical monitoring are also requirements to ensure that the systems are always operating within the requirement of Safety Instrument Functions (SIF) and Safety Integrity Level (SIL).

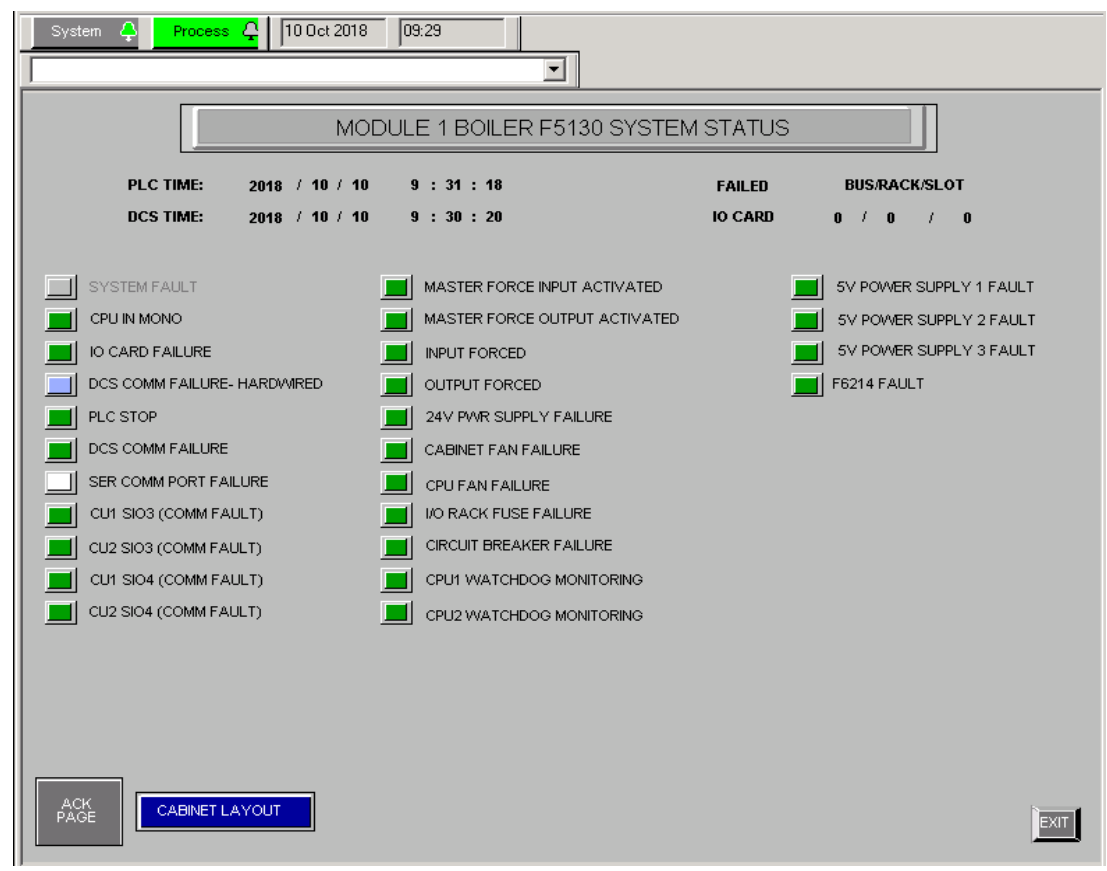

Fig. 13 - Boiler Management System Status Monitoring

\subsection{Proactive/Predictive Maintenance Methods for Final Elements}

A good performing sensor and system will not ensure good instrument loop operation if the final element is not performing well. Final elements that are typically used to control and bring the process to a safe state are control valves and shutdown valves. Performance of control valves using smart positioners provides valuable data and information regarding the performance of the valve internals, actuators and accessories. This was not possible during the pneumatic operating era whereby no diagnostic was provided. With the advancement of electronics and microprocessors capabilities, the availability of smart sensors applied to the control valve positioners allows for valuable information and data to be provided for analysis and proactive/predictive maintenance. Control valve manufacturers have developed diagnostic systems with a user interface that allows for the information received from the valve's positioner which 
helps users to understand the true health of these instruments. These software focus on the device health, providing information that allows for root cause identification, so rectification can be initiated and completed without multiple reworks. Having these tools provides the means to make correct and effective maintenance judgments. Control valves in the industry are classified to different criticality based on a risk matrix. The higher the criticality, the higher the impact in terms of economic loss, environment or health and safety.

Typically, conventional maintenance strategies focus on the higher critical valves with control valve overhaul being scheduled during the plant turnaround. Conventional maintenance is based on a fixed type with fixed materials being purchased. Typically for valves, soft parts are purchased as per recommended. This exercise causes significant economical lost as some of these valves could actually be in good condition and the operation could be extended. With this data now being available, analysis on the historical data allows for predicting the remaining lifespan or duration to failure. Figure 14 shows a sample of the dashboard for monitoring of control valve.

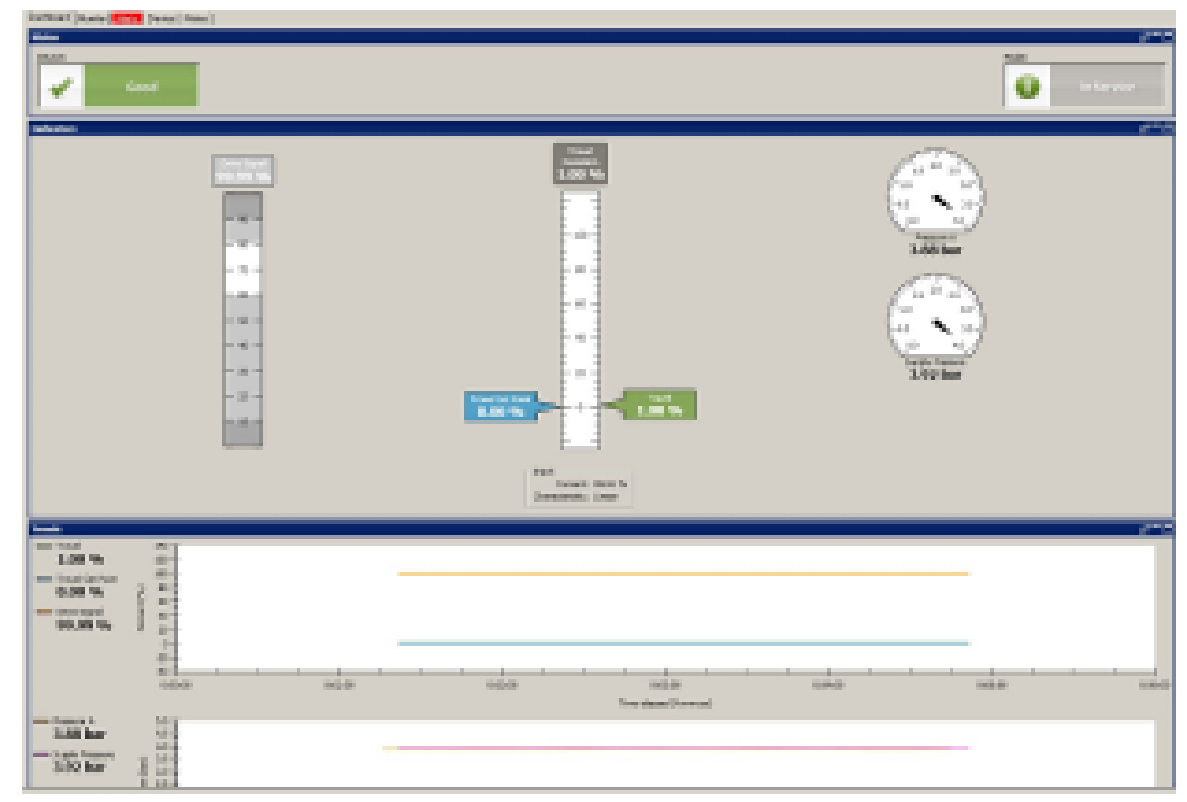

Fig. 14 - Dashboard Monitoring for Control Valve

\subsection{Proactive/Predictive Maintenance Methods for Process and Major Equipment}

Typical process plants have thousands of parameters that are continuously recording measurements from sensors that generate millions of data points every day. Through the application of machine learning with risk management criteria, the entire spectrum of the process data can be analysed and critical findings or patterns can be observed, which could be the precursors of potential failures. Application of these data into new infrastructures are made possible due to advancement in IT technologies such as the application of OPC, cloud and integration with OT.

\subsection{Centralize Monitoring and Dashboard}

With thousands of data and information being made available from the sensors installed in the process plant that are fed into the various systems, this can greatly overwhelm the users and making quick and correct actions on any deviations or failures. This has led to development of graphical overviews, centralized monitoring and dashboard concepts to provide situational awareness to the users. Example provided in Figure 15. The information provided is captured from the Asset Management System, with a database function developed in SQL that allows for tabulation of the data to user specific information. 


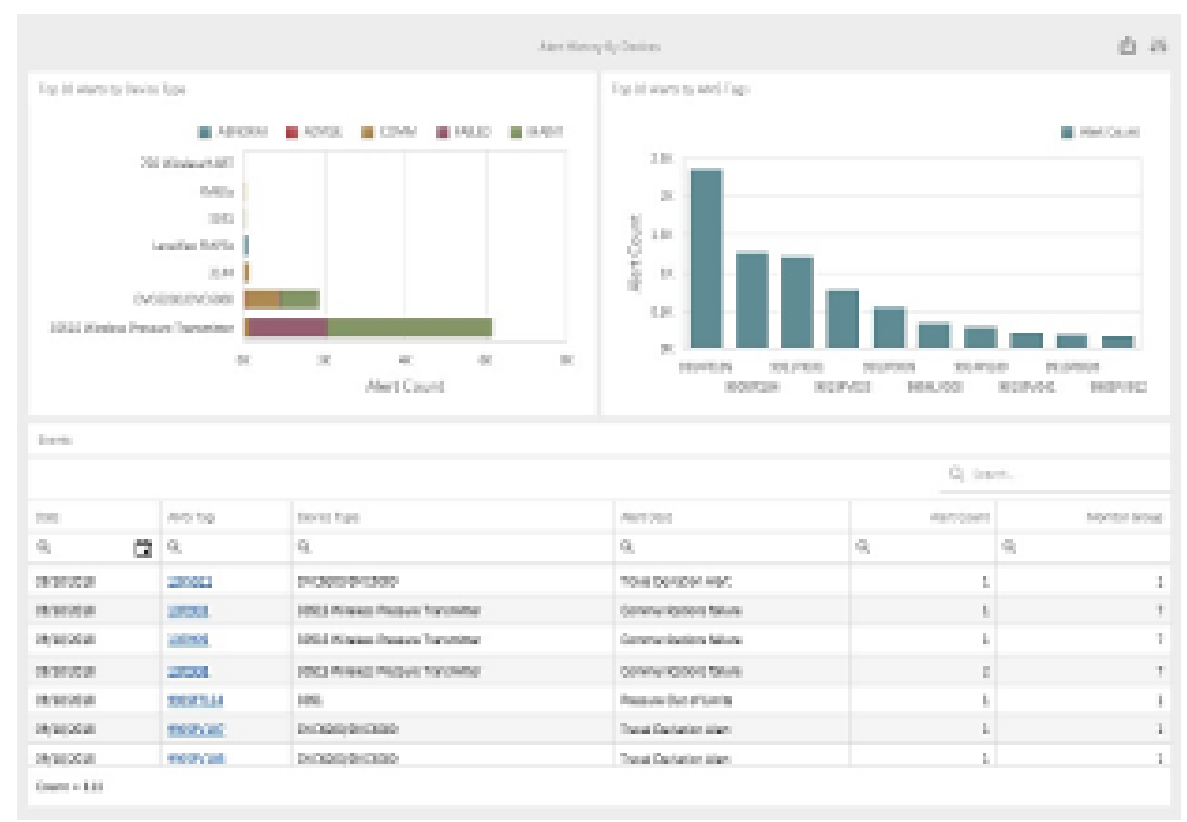

Fig. 15 - Dashboard Concept for Instrument Health Check

\subsection{Results and Discussion}

Application of trips to the process plant and reduced the downtime by the quicker turnaround and better troubleshooting and root cause identification. All safety critical equipment that is part of the onion layer barrier is maintained and assured as the information available is transparent. Table 1 tabulates the strength and weakness of the various predictive maintenance tools explained in the previous section.

\begin{tabular}{lll}
\hline \multicolumn{1}{c}{ Proactive/Predictive Tool } & \multicolumn{1}{c}{ Strength } & \multicolumn{1}{c}{ Weakness } \\
\hline Transmitter Deviation Alarm & $\begin{array}{l}\text { Provide continuous testing and alert } \\
\text { transmitter drift or no response. }\end{array}$ & $\begin{array}{l}\text { False alarm if transmitter have } \\
\text { different response time. }\end{array}$ \\
\hline Transmitter Fault Detection & $\begin{array}{l}\text { Utilization of transmitter mA signal to } \\
\text { provide transmitter health condition. }\end{array}$ & $\begin{array}{l}\text { Require configuration in systems for } \\
\text { detection and activation of alarm/alert. }\end{array}$ \\
\hline Control Valve Asset Monitoring & $\begin{array}{l}\text { Able to provide live data of control } \\
\text { valve such as friction, actuator } \\
\text { leakage etc. }\end{array}$ & $\begin{array}{l}\text { Require smart positioners that is prone } \\
\text { to electronic failures when installed in } \\
\text { harsh conditions. }\end{array}$ \\
\hline Transmitter Asset Monitoring & $\begin{array}{l}\text { Able to provide transmitter health } \\
\text { information such as operating } \\
\text { temperature, electronics health. }\end{array}$ & $\begin{array}{l}\text { Require smart transmitters that are } \\
\text { prone to electronics failure when } \\
\text { installed in harsh conditions. }\end{array}$ \\
\hline Systems Asset Monitoring & $\begin{array}{l}\text { Provide status of systems and } \\
\text { peripherals. }\end{array}$ & Fine tuning of diagnostics required. \\
\hline
\end{tabular}

Table 1 - Comparison of Various Proactive/Predictive Tools

\subsection{Conclusion}

Industrial plants should no longer assume that equipment failures will only occur after some fixed amount of time in service. For Instrumentation \& Control, it is important to ensure that the sensors, systems and final elements are working within the design limits to ensure the barriers for process safety is always available. Furthermore, these instrumentations also provide predictive analytics information for main equipment such as gas turbines, steam turbines, motors, compressors, heat exchangers and process conditions that are required to be in service and provide reliable information. With this in place, deployment of proactive/predictive and online maintenance can be applied.

\section{Acknowledgement}

This paper is fully supported by Malaysia LNG Sdn Bhd, A subsidiary of PETRONAS. The authors fully acknowledged Schneider Electric, Emerson, Flowserve, PI OSI and Universiti Teknologi Malaysia's Faculty of Electrical Engineering in the application of Instrument \& Control Proactive/Predictive Maintenance in the industry and the continuous development worked on. 


\section{References}

[1] Hogxia, W., Xiaohui, Y., and Ming, Y. 2016. Study on Predictive Maintenance Strategy. International Journal of $u$ - and e-Service, Science and Technology. 9(4):295-300.

[2] Hashemian, H.M. 2008. Predictive Maintenance of Critical Equipment in Industrial Processes.

[3] Sun, S. Research on Assessment and Prediction about the Reliability of Aircraft Engine Oriented Predictive Maintenance. 2013. Nanjing University of Aeronautics and Astronautics.

[4] Snooke, N., and Price, C. 2012. Automated FMEA- Based Symptom Generation. Advanced Engineering Informatics (26): 870-888.

[5] Hart, Douglas.2017. Implementing a "Best Practices" Predictive Maintenance Program: Avoiding the 10 Most Common Pitfalls. Emerson Reliability Consulting.

[6] Thakker, M. and Patel, M.J. 2016. Review on Instrumentation \& Safety System Techniques for Upstream Oil \& Gas Sector. Research Gate Publication.

[7] Hashemian, H.M. and Bean, W.C. 2011. State-of-the Art Predictive Maintenance Techniques. IEEE Transactions on Instrumentation and Measurement 60(10): 3480-3492.

[8] Hawkins, B. 2016. Instrumentation-It's Not Just for Process Control Anymore. Emerson Process Systems \& Solutions.

[9] Hashemian, H.M.2007. Accurately Measure the Dynamic Response of Pressure Instruments. Power. 72-78.

[10] Cristinel, N.D., Valentin, N. and Luminta, B. 2012. Theoretical Study about the Influence of Predictive Maintenance on Process Equipment Lifetime. Journal of Engineering Studies and Research. 18(2). 57-65.

[11] Despande, A., Pitale, P., and Sanap, S. 2016. Industrial Automation using Internet of Things (IOT). International Journal of Advanced Research in Computer Engineering \& Technology (IJARCET). 5(20). 266-269.

[12] Martin, P. 2017. Value-Focused Industrial Internet of Things - Just a Hype or a Real Business Value? Schneider Electric White Paper.

[13] Clary, T. and Diaz, M. 2018. The Unexpected Impact of IIoT-driven Modernization on Plant Operational Profitability. Schneider Electric White Paper.

[14] Martin, P.G. 2018. Improved Operational Profitability: Is IIoT a game changer in Asset Performance? Schneider Electric White Paper.

[15] Emerson-Fisher.2017. Valve-Link Software Signature Series Performance Testing. Product Bulletin.

[16] Emerson-Fisher. 2017. Valve-Link Software Manual. Product Bulletin.

[17] Flowserve. 2015. Valve-Sight Prevention Delivered. Product Bulletin.

[18] Invensys, Triconex. 2010. Triconex General Purpose v2 Systems- Safety Considerations Guide.

[19] HIMA.2011. HIMax ${ }^{\circledR}$ Safety Manual.

[20] Zorzi, M., Gluhak, A., Lange, S., and Bassi, A. 2010. From today's intranet of things to a future internet of things: a wireless-and mobility-related view. Wireless Communications, IEEE. 17(6). 44-51. 